Research article

Open Access

\title{
A polymorphism in the 3 ' untranslated region of the gene encoding prostaglandin endoperoxide synthase 2 is not associated with an increase in breast cancer risk: a nested case-control study
}

David G Cox ${ }^{1,2}$, Julie Buring ${ }^{3}$, Susan E Hankinson ${ }^{2,4}$ and David J Hunter ${ }^{1,2}$

\author{
${ }^{1}$ Program in Genetic and Molecular Epidemiology, Epidemiology Department, Harvard School of Public Health, 677 Huntington Avenue, Boston, MA \\ 02115, USA \\ ${ }^{2}$ Channing Laboratory, Department of Medicine, Brigham and Women's Hospital, Harvard Medical School, 181 Longwood Avenue, Boston, MA \\ 02115, USA \\ 3Division of Preventive Medicine, Department of Medicine, Brigham and Women's Hospital, Harvard Medical School, 900 Commonwealth Avenue, \\ Boston, MA 02215, USA \\ 4Epidemiology Department, Harvard School of Public Health, 677 Huntington Avenue, Boston, MA 02115, USA
}

Corresponding author: David G Cox, dcox@hsph.harvard.edu

Received: 26 Oct 2006 Revisions requested: 13 Nov 2006 Revisions received: 16 Nov 2006 Accepted: 10 Jan 2007 Published: 10 Jan 2007

Breast Cancer Research 2007, 9:R3 (doi:10.1186/bcr1635)

This article is online at: http://breast-cancer-research.com/content/9/1/R3

(C) 2007 Cox et al.; licensee BioMed Central Ltd.

This is an open access article distributed under the terms of the Creative Commons Attribution License (http://creativecommons.org/licenses/by/2.0), which permits unrestricted use, distribution, and reproduction in any medium, provided the original work is properly cited.

\begin{abstract}
Introduction Prostaglandins are integral components in the cellular response to inflammation, promoting cellular proliferation and angiogenesis. The enzyme responsible for the conversion of arachidonic acid to prostaglandins in response to inflammation is prostaglandin endoperoxide synthase 2/cyclooxygenase 2 (PTGS2/COX2). Polymorphisms in the PTGS2 gene have been associated with various diseases, including inflammatory bowel disease and cancer of the lung, colorectum, and breast.

Methods We genotyped the five most common polymorphisms (rs20417, rs5277, rs20432, rs5275, and rs4648298) in the Nurses' Health Study (1,270 cases, 1,762 controls) to test the hypothesis that polymorphisms in PTGS2 are associated with breast cancer risk, using logistic regression analyses. The Nurses' Health Study 2 (317 cases, 634 controls) and Harvard Women's Health Study (702 cases, 703 controls) were used to further examine putative associations.
\end{abstract}

Results The rs5275 polymorphism in the 3' untranslated region of the PTGS2 gene was associated with a decrease in breast cancer risk. We therefore genotyped this single-nucleotide polymorphism in the Nurses' Health Study 2 and Harvard Women's Health Study. Similar results were observed in these subsequent analyses, with no statistically significant heterogeneity in risk estimates between studies. In pooled analyses, women homozygous for the T allele at rs5275 had a $20 \%$ lower risk of breast cancer than those homozygous for the $\mathrm{C}$ allele (odds ratio $0.80,95 \%$ confidence interval 0.66 to 0.97 ).

Conclusion Although this polymorphism may be associated with a decrease in breast cancer risk among Caucasian women, we provide strong evidence that it is not associated with an increased risk of breast cancer.

\section{Introduction}

Inflammation is thought to have a role in many diseases, including cancers [1]. Cellular response to inflammation signaling can lead to increased expression of growth factors and their receptors, which in turn can lead to cellular proliferation [2]. In addition, by-products of the inflammatory response, such as free radicals, can cause DNA damage [3-5]. Taken together, these factors suggest a strong a priori hypothesis that inflammation is related to the etiology of cancer.

Although there is an inflammatory type of breast cancer, this accounts for only 1 to $5 \%$ of the overall incidence of breast

$\overline{\mathrm{AFB}}=$ age at first birth; COX2 = cyclo-oxygenase 2; NHS = Nurses' Health Study; PTGS2 = prostaglandin endoperoxide synthase 2; SNP = singlenucleotide polymorphism; UTR $=$ untranslated region; WHS $=$ Harvard Women's Health Study. 
cancer in the United States [6]. Non-steroidal anti-inflammatory drugs have been shown to be inversely associated with risk of colorectal cancer risk [7]. However, the association between these drugs and breast cancer risk is unclear $[8,9]$. Nevertheless, causal relationships between prostaglandin endoperoxide synthase 2/cyclo-oxygenase 2 (PTGS2/COX2) and mammary epithelial tumorigenesis have been detected in animal models [10].

PTGS2 is a protein that is expressed as part of the acute response to inflammation [11] and is the main enzyme responsible for transforming arachidonic acid into prostaglandins. Prostaglandins in turn stimulate cell proliferation and angiogenesis. Expression of PTGS2 is tightly controlled, with basal levels low to nonexistent in most tissue types. In addition, PTGS2 mRNA has a very short half-life regulated by sequence-specific elements in the $3^{\prime}$ untranslated region (UTR) of the gene $[12,13]$.

We hypothesize that polymorphisms in this gene could influence the risk of breast cancer, through altering the levels of expression or activity of the PTGS2 enzyme. We have therefore genotyped five single-nucleotide polymorphisms (SNPs) in the PTGS2 gene in the breast cancer cases and controls nested within the Nurses' Health Study (NHS). One of these polymorphisms was statistically associated with decreased breast cancer risk, and we therefore sought to confirm this association in the breast cancer cases and controls nested within the Nurses' Health Study 2 (NHS2) and Harvard Women's Health Study (WHS).

\section{Materials and methods}

The NHS nested breast cancer case-control study (cases, $n=$ 1,270 ; controls, $n=1,762$ ) is derived from 32,826 women who were free of diagnosed breast cancer at blood collection in 1989 and 1990, since when they were followed for incident disease until 31 May 2000. Medical records were used to confirm the diagnoses in women who reported a diagnosis of breast cancer on the biennial questionnaires. Control subjects were matched to cases on the basis of age, menopausal sta- tus, recent hormone replacement therapy, and blood-draw specific variables (such as date and time of day). The NHS2 breast cancer case-control study (cases, $n=317$; controls, $n$ $=634$ ) is nested in a study of 29,611 women who were free of diagnosed breast cancer at the time of blood draw (between 1996 and 1999) and were followed for incident disease until 1 June 2003. The nested breast cancer case-control study in the WHS began in 1993, when 28,263 women provided blood samples and were followed for incident disease until 7 March 2000, with 702 cases and 703 controls. Cases and controls were selected for the NHS2 and WHS with the use of the same criteria as in the NHS. All three cohorts use similar questionnaires to collect covariate information, administered biennially. Detailed descriptions of these three cohorts have been published previously [14-16]. Informed consent was obtained from all participants, and the study was approved by the Institutional Review Board of the Brigham and Women's Hospital.

PTGS2 is a very small gene (less than 10 kilobase pairs from 5' UTR to 3' UTR), with only five known common polymorphisms; linkage disequilibrium between them is high (rs20417, rs5277, rs20432, rs5275, rs4648298 [17,18]; see Table 1 for more information). These five SNPs were genotyped by means of the 5' nuclease assay (TaqMan; Applied Biosystems, Foster City, CA, USA). The SNPs are located at base pairs $926,3,050,5,209,8,473$, and 9,850 on GenBank sequence D28235. rs20417 is in the $5^{\prime}$ region, rs5277 is a synonymous polymorphism in exon 3 , rs20432 is in the intron between exons 5 and 6 , and both rs5275 and rs4648298 are in the 3' UTR of PTGS2. TaqMan primers, probes, and conditions for genotyping assays are available from the authors on request. All genotyping was done with laboratory personnel blinded to the case-control status of the samples and included quality-control samples (DNA from cohort participants, not part of the case-control study, repeated from 2 to 18 times across plates) for validation. Concordance for quality-control samples was $100 \%$.

Statistical analysis was performed with SAS version 9.1 (SAS

Table 1

Pairwise linkage disequilibrium between SNPs selected for study in PTGS2

\begin{tabular}{llllll}
\hline SNP & PTGS2_20417 & PTGS2_5277 & PTGS2_5209 & PTGS2_5275 & PTGS2_4648298 \\
\hline PTGS2_20417 & - & 1 & 0.97 & 0.95 & 0.98 \\
PTGS2_5277 & 0.03 & - & 1 & 1 & 0.84 \\
PTGS2_5209 & 0.13 & 0.03 & - & 1 & 1 \\
PTGS2_5275 & 0.1 & 0.06 & 0.11 & - & 1 \\
PTGS2_4648298 & 0.01 & 0.002 & 0.02 & 0.01 & -
\end{tabular}

Numbers above the diagonal are the Lewontin's $D^{\prime}$, a measure of linkage disequilibrium; numbers under the diagonal are the correlation coefficient $r$. PTGS2, prostaglandin endoperoxide synthase 2; SNP, single-nucleotide polymorphism. 
Institute, Cary, NC, USA). Haplotype estimation was performed with PROC HAPLOTYPE, tests for deviation from Hardy-Weinberg equilibrium were performed with PROC ALLELE, and haplotype-specific odds ratios and 95\% confidence intervals were calculated with unconditional logistic regression with PROC LOGISTIC, controlling for age (at blood draw as a continuous variable), age at first birth (AFB), parity (nulliparous; one or two children and AFB 24 years or less; one or two children and AFB more than 24 years; more than two children and AFB 24 years or less; more than two children and AFB more than 24 years), menopausal status at diagnosis (premenopausal, postmenopausal, unknown), history of benign breast disease (yes/no), and family history of breast cancer (yes/no). Variables such as AFB, history of benign breast disease, or family history of breast cancer are updated in each questionnaire cycle, and the variable closest to the diagnosis (cases) or index date (controls) is used in these analyses. Cis-interactions, comparing associations with haplotypes to those observed with independent SNPs, were tested by comparing the model with the SNP genotype alone to the model with each haplotype carrying the risk allele by means of likelihood ratio testing. Interaction $p$ values were cal- culated by likelihood ratio testing, comparing the model with main effects for each exposure (polymorphism and anti-inflammatory use) to the model including the cross-tabulation of the two exposures. Heterogeneity between cohorts was calculated with Cochran-Mantel-Haenszel statistics in PROC FREQ.

\section{Results}

We did not detect any deviation from Hardy-Weinberg equilibrium among the controls in the NHS at any of the polymorphisms studied (data not shown). Only the rs5275 polymorphism in the 3' UTR of the PTGS2 gene showed an association with risk of breast cancer (Table 2) and was therefore subsequently genotyped in the NHS2 and WHS. There is almost complete linkage disequilibrium (Lewontin $D^{\prime}=0.95$ ) between the rs20417 polymorphism 5' of PTGS2 and rs5275 3' of PTGS2. Similarly, one PTGS2 haplotype carrying the variant allele of rs5275 was associated with a decrease in breast cancer risk. However, the global test for difference in haplotype frequencies between breast cancer cases and controls was not significant ( $p=0.15$; Table 3). Testing for cis-interac-

Table 2

Association between PTGS2 genotypes and breast cancer risk in the Nurses' Health Study

\begin{tabular}{|c|c|c|c|c|}
\hline SNP & Genotype & Cases (percentage) & Controls (percentage) & OR $(95 \% \mathrm{Cl})^{\mathrm{a}}$ \\
\hline \multirow[t]{4}{*}{ PTGS2_20417 } & $\mathrm{G} / \mathrm{G}$ & $865(69.6)$ & $1,185(69.1)$ & 1.00 (reference) \\
\hline & $\mathrm{G} / \mathrm{C}$ & $336(27.0)$ & $485(28.3)$ & $0.93(0.78-1.10)$ \\
\hline & $\mathrm{C} / \mathrm{C}$ & $42(3.4)$ & $45(2.6)$ & $1.28(0.82-2.02)$ \\
\hline & & & & $p$ trend $=0.94$ \\
\hline \multirow[t]{4}{*}{ PTGS2_5277 } & $\mathrm{G} / \mathrm{G}$ & $875(71.0)$ & $1,217(71.0)$ & 1.00 (reference) \\
\hline & $\mathrm{G} / \mathrm{C}$ & $322(26.1)$ & $464(27.1)$ & $1.00(0.84-1.19)$ \\
\hline & $\mathrm{C} / \mathrm{C}$ & $35(2.8)$ & $34(2.0)$ & $1.35(0.81-2.26)$ \\
\hline & & & & $p$ trend $=0.55$ \\
\hline \multirow[t]{4}{*}{ PTGS2_5209 } & $\mathrm{T} / \mathrm{T}$ & $834(69.4)$ & $1,171(69.5)$ & 1.00 (reference) \\
\hline & $\mathrm{T} / \mathrm{G}$ & $330(27.5)$ & $472(28.0)$ & $0.97(0.82-1.16)$ \\
\hline & $\mathrm{G} / \mathrm{G}$ & $38(3.2)$ & $43(2.6)$ & $1.24(0.77-2.00)$ \\
\hline & & & & $p$ trend $=0.81$ \\
\hline \multirow[t]{4}{*}{ PTGS2_5275 } & $\mathrm{T} / \mathrm{T}$ & 541 (43.3) & $699(40.6)$ & 1.00 (reference) \\
\hline & $\mathrm{T} / \mathrm{C}$ & $567(45.4)$ & $808(47.0)$ & $0.88(0.75-1.04)$ \\
\hline & $\mathrm{C} / \mathrm{C}$ & $141(11.3)$ & $213(12.4)$ & $0.80(0.62-1.03)$ \\
\hline & & & & $p$ trend $=0.05$ \\
\hline \multirow[t]{2}{*}{ PTGS2_4648298 } & $\mathrm{A} / \mathrm{A}$ & $1,180(95.4)$ & 1,654 (96.3) & 1.00 (reference) \\
\hline & $A / G+G / G$ & $57(4.6)$ & $63(3.7)$ & $1.29(0.88-1.88)$ \\
\hline
\end{tabular}

aUnconditional logistic regression controlled for age, BMl at age 18 years, menopausal status at diagnosis, history of benign breast disease, and family history of breast cancer. $\mathrm{Cl}$, confidence interval; PTGS2, prostaglandin endoperoxide synthase 2; OR, odds ratio; SNP, single-nucleotide polymorphism. 
Breast Cancer Research Vol 9 No 1 Cox et al.

Table 3

PTGS2 haplotypes and breast cancer risk in the Nurses' Health Study

\begin{tabular}{llll}
\hline Haplotype & Cases (percentage) & Controls (percentage) & OR (95\% Cl)a \\
\hline z1 G-G-T-T-A & $1,250(49.4)$ & $1,691(48.2)$ & $1.00($ reference) \\
z2 G-G-T-C-A & $435(17.2)$ & $667(19.0)$ & $0.84(0.72-0.98)$ \\
z3 G-C-T-T-A & $403(15.9)$ & $544(15.5)$ & $0.99(0.85-1.17)$ \\
z4 C-G-G-C-A & $358(14.1)$ & $507(14.5)$ & $0.92(0.79-1.09)$ \\
z5 C-G-G-C-G & $53(2.1)$ & $63(1.8)$ & $1.16(0.78-1.72)$ \\
\end{tabular}

aUnconditional logistic regression controlled for age, BMl at age 18 years, menopausal status at diagnosis, history of benign breast disease, and family history of breast cancer. $\mathrm{Cl}$, confidence interval; OR, odds ratio; PTGS2, prostaglandin endoperoxide synthase 2.

tion confirmed that this haplotype was not more strongly associated with breast cancer risk than the rs 5275 polymorphism alone. No difference in genotype distribution of rs5275 was observed between tumors positive or negative for sex steroid hormone receptors, no significant difference in risk was observed upon stratification by menopausal status, and no statistically significant interactions were detected between this polymorphism and use of aspirin or other non-steroidal antiinflammatory drugs at baseline (evaluated only in the NHS; data not shown).
Similar genotype distributions were observed among both the NHS2 and WHS with respect to the NHS1 at rs5275. On pooling the three data sets (Table 4), no heterogeneity among the studies was detected ( $p=0.12)$, and the pooled risk estimate shows a moderately statistically significant trend of decreasing risk with each variant rs5275 allele (odds ratio $0.91,95 \%$ confidence interval 0.83 to $0.99, p=0.02$ ).

Table 4

PTGS2 rs5275 genotype and breast cancer risk within the NHS1, NHS2, WHS, and pooled data

\begin{tabular}{|c|c|c|c|c|}
\hline Study & Genotype & Cases (percentage) & Controls (percentage) & OR $(95 \% \mathrm{Cl})^{a}$ \\
\hline \multirow[t]{4}{*}{ NHS1 } & $\mathrm{T} / \mathrm{T}$ & $541(43.3)$ & $699(40.6)$ & 1.00 (reference) \\
\hline & $\mathrm{T} / \mathrm{C}$ & $567(45.4)$ & $808(47.0)$ & $0.89(0.75-1.04)$ \\
\hline & $\mathrm{C} / \mathrm{C}$ & $141(11.3)$ & $213(12.4)$ & $0.80(0.62-1.03)$ \\
\hline & & & & $p$ trend $=0.05$ \\
\hline \multirow[t]{4}{*}{$\mathrm{NHS} 2$} & $\mathrm{~T} / \mathrm{T}$ & $140(46.5)$ & $270(44.3)$ & 1.00 (reference) \\
\hline & T/C & $131(43.5)$ & $259(42.5)$ & $0.97(0.72-1.31)$ \\
\hline & $\mathrm{C} / \mathrm{C}$ & $30(10.0)$ & 81 (13.3) & $0.72(0.45-1.15)$ \\
\hline & & & & $p$ trend $=0.26$ \\
\hline \multirow[t]{4}{*}{ WHS } & $\mathrm{T} / \mathrm{T}$ & $281(43.6)$ & $278(42.7)$ & 1.00 (reference) \\
\hline & $\mathrm{T} / \mathrm{C}$ & $296(46.0)$ & $294(45.2)$ & $0.99(0.78-1.26)$ \\
\hline & $\mathrm{C} / \mathrm{C}$ & $67(10.4)$ & $79(12.1)$ & $0.86(0.58-1.25)$ \\
\hline & & & & $p$ trend $=0.53$ \\
\hline \multirow[t]{4}{*}{ Pooled } & $\mathrm{T} / \mathrm{T}$ & $962(43.8)$ & $1,246(41.8)$ & 1.00 (reference) \\
\hline & $\mathrm{T} / \mathrm{C}$ & $994(45.3)$ & $1,361(45.7)$ & $0.93(0.82-1.05)$ \\
\hline & $\mathrm{C} / \mathrm{C}$ & $238(10.8)$ & $373(12.5)$ & $0.80(0.66-0.97)$ \\
\hline & & & & $p$ trend $=0.02$ \\
\hline
\end{tabular}

aUnconditional logistic regression controlled for age, age at first birth and parity, menopausal status at diagnosis, history of benign breast disease, and family history of breast cancer. Cl, confidence interval; NHS, Nurses' Health Study; OR, odds ratio; PTGS2, prostaglandin endoperoxide synthase 2; WHS, Harvard Women's Health Study. 


\section{Discussion}

Variant expression or activity of PTGS2 could alter the risk of breast neoplasia, independently of exposure to aspirin. Prostaglandins stimulate cell migration and lead to proliferation and neovascularization [2]. Byproducts of the peroxidase activity of PTGS2 are free radicals, which are known mutagens [3-5]. Last, prostaglandin $E_{2}$ induces aromatase, the main enzyme in the production of estrogen [19].

Recently Langsenlehner and colleagues published an association between the rs5275 SNP and breast cancer risk [20]. They observed a doubling in risk with the $\mathrm{C} / \mathrm{C}$ genotype of this SNP in comparison with the T/T genotype. Interestingly, this polymorphism is not in Hardy-Weinberg equilibrium in either the case or the control group of the study presented by Langsenlehner and colleagues. Although the statistical significance of an inverse association between rs5275 and breast cancer risk among Caucasians (all three studies presented are more than $98 \%$ Caucasian in origin) as presented here requires replication, we can comfortably exclude a modest increase in risk associated with this genotype.

No in vitro or in vivo information regarding any putative change in function associated with this polymorphism is available. Given its location in the 3' UTR, the rs5275 polymorphism is a likely candidate to influence mRNA half-life, which is controlled by sequence-specific elements in this region of the mRNA [21]. It is possible that the rs5275 SNP is not a functional variant but a marker in linkage disequilibrium with variant(s) that alter breast cancer risk. Although linkage disequilibrium is strong across the PTGS2 gene, differences in the degree and extent of linkage disequilibrium between populations may explain variation in risk estimates from one study to another. Linkage between the rs5275 SNP and a causal variant would also explain why the strongest association with breast cancer risk is seen with this polymorphism and not the others. Examination of how the PTGS2 enzyme works in the breast, and in particular neoplastic breast cells, could shed light on its relation to breast cancer risk.

\section{Conclusion}

Although polymorphisms, particularly the rs5275 polymorphism in the 3' UTR, of PTGS2 may be associated with a decrease in breast cancer risk, we provide strong evidence that it is not associated with an increased risk of breast cancer.

\section{Competing interests}

The authors declare that they have no competing interests.

\section{Authors' contributions}

DGC performed analyses and prepared the manuscript. JB, $\mathrm{SEH}$, and $\mathrm{DJH}$ were responsible for sample collection, editing the manuscript, and securing funding for the study. All authors read and approved the final manuscript.

\section{Acknowledgements}

We are indebted to the participants in the NHS1, NHS2, and WHS for their continuing dedication and commitment. We thank the laboratory of Patrice Soule for DNA extraction and plating, and Hardeep Ranu for TaqMan genotyping. This study was supported by National Institutes of Health research grants CA87969, CA49449, and CA65725. DGC is supported by training grant CA 09001-27 from the National Institutes of Health.

\section{References}

1. Hofseth $L J$, Ying $L$ : Identifying and defusing weapons of mass inflammation in carcinogenesis. Biochim Biophys Acta 2006, 1765:74-84.

2. Backlund MG, Mann JR, Dubois RN: Mechanisms for the prevention of gastrointestinal cancer: the role of prostaglandin E2. Oncology 2005, 69(Suppl 1):28-32.

3. Lee SH, Williams MV, Dubois RN, Blair IA: Cyclooxygenase-2mediated DNA damage. J Biol Chem 2005, 280:28337-28346.

4. Williams MV, Lee SH, Pollack M, Blair IA: Endogenous lipid hydroperoxide-mediated DNA-adduct formation in min mice. $J$ Biol Chem 2006, 281:10127-10133.

5. Blair IA: Lipid hydroperoxide-mediated DNA damage. Exp Geronto/ 2001, 36:1473-1481.

6. Walshe JM, Swain SM: Clinical aspects of inflammatory breast cancer. Breast Dis 2005, 22:35-44.

7. Raju R, Cruz-Correa M: Chemoprevention of colorectal cancer. Dis Colon Rectum 2006, 49:113-124.

8. Egan KM, Stampfer MJ, Giovannucci E, Rosner BA, Colditz GA: Prospective study of regular aspirin use and the risk of breast cancer. J Natl Cancer Inst 1996, 88:988-993.

9. Harris RE, Chlebowski RT, Jackson RD, Frid DJ, Ascenseo JL, Anderson G, Loar A, Rodabough RJ, White E, McTiernan A: Breast cancer and nonsteroidal anti-inflammatory drugs: prospective results from the Women's Health Initiative. Cancer Res 2003, 63:6096-6101.

10. Sinicrope FA: Targeting cyclooxygenase-2 for prevention and therapy of colorectal cancer. Mol Carcinog 2006, 45:447-454.

11. Martin Sanz P, Hortelano S, Bosca L, Casado M: Cyclooxygenase 2: understanding the pathophysiological role through genetically altered mouse models. Front Biosci 2006, 11:2876-2888.

12. Lee HK, Jeong S: Beta-catenin stabilizes cyclooxygenase-2 mRNA by interacting with AU-rich elements of 3'-UTR. Nucleic Acids Res 2006, 34:5705-5714.

13. Dixon DA: Dysregulated post-transcriptional control of COX-2 gene expression in cancer. Curr Pharm Des 2004, 10:635-646.

14. Rockhill B, Willett WC, Hunter DJ, Manson JE, Hankinson SE, Spiegelman D, Colditz GA: Physical activity and breast cancer risk in a cohort of young women. J Natl Cancer Inst 1998, 90:1155-1160.

15. Rexrode KM, Lee IM, Cook NR, Hennekens CH, Buring JE: Baseline characteristics of participants in the Women's Health Study. J Womens Health Gend Based Med 2000, 9:19-27.

16. Hankinson SE, Willett WC, Manson JE, Colditz GA, Hunter DJ, Spiegelman D, Barbieri RL, Speizer FE: Plasma sex steroid hormone levels and risk of breast cancer in postmenopausal women. J Natl Cancer Inst 1998, 90:1292-1299.

17. Halushka MK, Fan JB, Bentley K, Hsie L, Shen N, Weder A, Cooper $R$, Lipshutz R, Chakravarti A: Patterns of single-nucleotide polymorphisms in candidate genes for blood-pressure homeostasis. Nat Genet 1999, 22:239-247.

18. Cox D, Boillot C, Canzian F: Data mining: efficiency of using sequence databases for polymorphism discovery. Hum Mutat 2001, 17:141-150

19. Brueggemeier RW, Richards JA, Petrel TA: Aromatase and cyclooxygenases: enzymes in breast cancer. J Steroid Biochem Mol Biol 2003, 86:501-507.

20. Langsenlehner U, Yazdani-Biuki B, Eder T, Renner W, Wascher TC, Paulweber B, Weitzer W, Samonigg H, Krippl P: The cyclooxygenase-2 (PTGS2) 8473T $>C$ polymorphism is associated with breast cancer risk. Clin Cancer Res 2006, 12:1392-1394.

21. Zhang Z, Sheng H, Shao J, Beauchamp RD, DuBois RN: Posttranscriptional regulation of cyclooxygenase-2 in rat intestinal epithelial cells. Neoplasia 2000, 2:523-530. 